\title{
Research
}

\section{General practice consultation rates for mothers and babies in the first year after birth according to place of birth. A descriptive study in one general practice}

Pat Hoddinott Highlands and Islands Health Research Institute, University of Aberdeen, UK; Julie A. Simpson Department of General Practice and Primary Care, University of Aberdeen, Aberdeen, UK and Roisin Pill Department of General Practice, University of Wales College of Medicine, Cardiff, UK

Maternal and infant health in primary care following childbirth is an important area which has received little research attention. Very little is known about primary health service use following childbirth and its determinants. The aim of this exploratory study was to test the hypothesis that place of birth is independently associated with mother and baby consultation patterns in the first year of life. This is a descriptive study of 453 pregnancies of 338 mothers from an inner city general practice between 1987 and 1996. There were 86 intended home births, 126 intended domino births and 240 intended hospital births, with one woman unbooked. Of these, 69 women had home births, 97 had domino births, 286 women had hospital deliveries and one baby was born in an ambulance. The outcome of interest was mother and baby consultation rates in the year following birth. In an attempt to control for pre-existing ill health we also calculated the women's consultation rate in the year prior to conception and subtracted this from the mother and baby consultation rate in the year following birth. We analysed the data according to both intended and actual place of birth. Women intending a home birth had fewer mother and baby consultations in the year following birth compared to women intending domino births, who in turn had fewer consultations than women intending hospital care during labour $(P<0.001)$. This gradient persisted when pre-existing maternal ill health was accounted for. A similar gradient of increasing consultation rates through home births, domino birth, normal vaginal delivery in hospital and assisted delivery in hospital was demonstrated when data were analysed according to actual place of birth. Other maternal characteristics independently associated with increased mother and baby consultation patterns were: primiparity, manual occupational class, not breastfeeding, maternal smoking, infertility prior to pregnancy, and recorded anxiety, depression or life events before or after birth. Women intending and having a home birth were older $(P<0.001)$ and less likely to be primiparous $(P=0.055)$ or of manual occupational class $(P<0.001)$. Babies born at home were heavier $(P<0.001)$, and women experienced less perineal trauma $(P<0.001)$ and were more likely to breastfeed at birth $(P=0.001)$. The conclusion we draw from this cohort of births, is that there is an association between women intending a home birth and women who actually have home births and lower mother and baby consultation rates postnatally, when compared with women having normal vaginal domino or hospital births. This is independent of maternal age, parity, smoking during pregnancy, occupational class, a history of infertility or miscarriage in the year prior to conception, anxiety, depression or life events in the year prior to conception or after birth, breastfeeding, sex of baby and birth weight.

Key words: consultation rates; home birth; postnatal health; primary care; breastfeeding 


\section{Introduction}

The incidence of home birth in England has increased from an all time low of $0.9 \%$ in 1985 to $2.2 \%$ in 1998 (Macfarlane and Mugford, 2000). Perinatal outcomes for home births have been reported (Chamberlain et al., 1996; Davies et al., 1996), but little is known about the implications of place of birth or method of delivery for consultation rates in general practice or for longerterm health outcomes. This is surprising given that considerable morbidity occurs after childbirth (Glazener et al., 1995; MacArthur etal., 1991). Data are available for the conditions which mothers and babies present in general practice in the first year after birth (Macfarlane and Mugford 2000), for women of child bearing age, and children aged 0-4 years linked to occupational class through the morbidity statistics from general practice (Royal College of General Practitioners, Office of Population Censuses and Surveys, and Department of Health, 1995). However, there are no data linking consultation patterns to place of birth. Factors found to be positively associated with higher consultation rates are: parents in manual occupations (Carlisle and Johnstone, 1998; Saxena et al., 1999), male children, (Royal College of General Practitioners, Office of Population Censuses and Surveys, and Department of Health, 1995) and being breastfed (personal communication, C Glazener, November 2000). However, it is unclear whether first-born children consult more than later born children (Bruijnzeels et al., 1998; Van Den Bosch et al., 1993).

This paper reports data from one inner city general practice, which had an unusually high number of home births compared with the national average. The aim was to test the hypothesis that place of birth is independently associated with mother and baby consultation patterns in the first year of life.

\section{Background and context}

The study was conducted in a general practice in the East End of London with high deprivation

Address for correspondence: Dr Pat Hoddinott, Macduff Medical Practice, 100 Duff Street, Macduff, Banffshire, AB44 1PR, UK. Email: hoddinott@doctors.org.uk
(Carstairs and Morris deprivation categories of 6 and 7) (Carstairs and Morris, 1989), but varied occupational class structure. There is considerable ethnic diversity, mostly Bengali, Black African, Black Caribbean and other Asian groups. A small community hospital maternity unit which was very popular with women because of its homely atmosphere and relatively low intervention rates closed just before this study commenced. The closest geographical maternity unit was a tertiary referral unit caring for some complicated pregnancies from outside the health authority area. Alternatively women could choose a large district general hospital maternity unit in a neighbouring borough, but with different midwifery teams and hence a reduction in continuity of care.

At the beginning of the study in May 1987, the practice had five female partners, a registrar and a part-time practice nurse. This became a six partner (four female, two male) practice with a registrar, assistant and two full-time practice nurses in 1996, the final year of the study. In 1993, the practice moved from small, overcrowded premises with community midwives based in the hospital to a purpose-built spacious medical centre, with attached, on-site health visitors and midwives. Historically the practice has provided mothers with community-based antenatal and intra-partum care. During the study period, the practice was unusual in having a relatively high proportion of mothers requesting a home birth compared with the national average. At confirmation of pregnancy and at antenatal booking, choices about antenatal care, labour care and place of birth were discussed and an antenatal pack containing written information about options for care was provided. Choices were flexible and could be changed at any time, including during labour. The majority of pregnant mothers in the practice chose community-based antenatal care, where antenatal booking was in the community and was arranged by two GP-attached midwives from a team of seven. This team of seven midwives covered a defined geographical area which included several GP practices. Antenatal care was then shared between the two midwives and the woman's general practitioner. A few mothers with complicated medical histories, for example diabetes, had some or all of their antenatal care with the obstetrician at the hospital. Mothers 
receiving community-based antenatal care could choose between a hospital birth, the domino scheme or a home birth. With the domino scheme, mothers were assessed and cared for at home by community midwives during early labour, before transferring to hospital when birth was imminent. With a hospital birth, mothers attended the hospital labour ward at the start of labour and were cared for by hospital midwives. A consultant obstetrician visited the practice monthly and saw mothers booked for a hospital birth at least once during the pregnancy. Mothers who booked for home or domino births only saw the consultant if a problem arose or at their request. Where possible the same two midwives attended the practice ante-natal clinic. Intra-partum care was provided by a rota of the seven midwives in the team and continuity of care was maximised where possible. Women had opportunities to meet all members of the team at antenatal classes. After birth all mothers had the option of a 6 hour discharge, with the GP visiting to perform the routine new-born examination either in hospital or at home.

The pattern of antenatal care provision over the study period did not change; however, intra-partum care did change. Between May 1987 and March 1994, a weekly rota of five GPs from different practices attended home and domino births with the midwife. The woman's own GP attended where possible, with the rota GP acting as back-up. Due to workload pressures, increased demand for home and domino births and the increasing autonomy of the community midwives, GPs stopped attending domino births in September 1993 and home births in March 1994. During the final year of this study, community-based teams of midwives were expanded to cover hospital births, as well as domino and home births. The midwifery teams became larger. The two midwives providing practice-based ante-natal care continued. There was less likelihood of continuity of care for women having home and domino births, but more likelihood of continuity of care for women having hospital births.

\section{Subjects and exclusion criteria for the study}

In May 1987 a maternity register was started at antenatal booking to record intended place of birth and birth outcomes. The register was maintained by one clerk throughout the study. She had sole responsibility for organising antenatal, postnatal and child health clinics and had considerable knowledge of childbirth in the practice. Birth outcome data were taken from the computerised obstetric summary, which was completed by the attendant midwife on the labour ward. The reasons for transfer from intended home birth to a domino or hospital care birth or from an intended domino birth to a hospital care birth were recorded in the maternity register by $\mathrm{PH}$ who was the GP providing intra-partum care. By January 1996, the total number of births after 24 completed weeks of pregnancy was 1021 . Of these 453 (44\%) births of 338 mothers were considered eligible for this study because there were no exceptional birth or perinatal complications and GP records were available for both the year preceding conception and following birth (Table 1). The following 568 of the 1021 mothers and babies in the register were excluded from the study: 514 mothers had either left the practice within a year of birth, had not been regis-

Table 1 Demographic data for births included in the study and births excluded

\begin{tabular}{|c|c|c|c|}
\hline & $\begin{array}{l}\text { Births } \\
\text { included } \\
(n=453)\end{array}$ & $\begin{array}{l}\text { Births } \\
\text { excluded } \\
\text { ( } n=568)\end{array}$ & $P$ value \\
\hline $\begin{array}{l}\text { Mean maternal age, } \\
\text { years (SD) (range) }\end{array}$ & $\begin{array}{l}29.2(5.3) \\
(16-42)\end{array}$ & $\begin{array}{l}27.1(5.7) \\
(16-49)\end{array}$ & $<0.001^{\mathrm{a}}$ \\
\hline Primiparous $n(\%)$ & $163(36.0)$ & $288(50.7)$ & $<0.001^{\mathrm{b}}$ \\
\hline $\begin{array}{l}\text { White } \\
\text { Other }\end{array}$ & $381(84.1)$ & $492(86.6)$ & $0.296^{b}$ \\
\hline Type of birth $n(\%)$ & & & \\
\hline $\begin{array}{l}\text { SVD home } \\
\text { SVD domino } \\
\text { SVD hospital } \\
\text { Assisted hospital } \\
\text { birth }\end{array}$ & $\begin{array}{r}69(15.2) \\
97(21.4) \\
201(44.4) \\
86(19.0)\end{array}$ & $\begin{array}{r}26(4.6) \\
95(16.7) \\
368(64.8) \\
79(13.9)\end{array}$ & $<0.001^{\mathrm{b}}$ \\
\hline $\begin{array}{l}\text { Deprivation category } \\
\quad 6\end{array}$ & $126(27.8)$ & \multirow[t]{2}{*}{$\begin{array}{l}\text { Not } \\
\text { recorded }\end{array}$} & \\
\hline $\begin{array}{l}7 \\
\text { Unknown } \\
\text { Occupational class } \\
n(\%)\end{array}$ & $\begin{array}{c}318(70.2) \\
9(2.0)\end{array}$ & & \\
\hline Manual & $253(55.8)$ & \multirow[t]{2}{*}{$\begin{array}{l}\text { Not } \\
\text { recorded }\end{array}$} & \\
\hline $\begin{array}{l}\text { Non-manual } \\
\text { Unknown }\end{array}$ & $\begin{array}{c}195(43.0) \\
5(1.1)\end{array}$ & & \\
\hline
\end{tabular}

Assisted hospital births includes forceps, ventouse and caesarian births.

SVD, spontaneous vaginal delivery.

aStudent's $t$ test.

${ }^{\mathrm{b}}$ Chi-squared test. 
tered for a year preceding conception or were away from their registered address for more than six weeks during the year preceding conception or the year after birth; 29 mothers had premature babies born before 36 completed weeks gestation; six mothers had babies with severe congenital abnormalities (Down's syndrome, cerebral palsy, haemophilia); nine mothers had stillborn babies or babies who died in the first year of life; eight mothers had twins; there was one maternal death and one mother was in a psychiatric hospital for 3 months.

\section{Consultation data: calculation of rates}

The combined mother and baby consultation rate in the year following birth (CMBFB) was calculated from GP records. As with other studies (Gunn et al., 1996), we have assumed that for each birth, a mother and her baby do not consult the GP independently of each other in the first year of life. For example, a mother with postnatal depression may present to the GP with minor anxieties about the babies' health. We have therefore used the sum of general practice consultations for mother and baby. In an attempt to control for pre-existing ill-health in the mother, the consultation rate for the year preceding conception was also calculated using the last menstrual period (LMP) as the end point. If this was uncertain, the dating scan was used to calculate the LMP. We have produced two measures of the impact of having a baby on mother and baby consultation rate. Firstly, the difference between the combined consultation rate for the year following birth minus the year preceding conception (DCMBFB) was calculated. Secondly, to aid interpretation we calculated the number of postnatal mother and baby consultations as a percentage of the total mother and baby consultations (PCMBFB).

$\mathrm{PCMBFB}=$

\section{CMBFB}

(mothers consultations pre-conception $+\mathrm{CMBFB})$ $\times 100$

If another child was conceived in the year preconception or post-birth, the consultation rate while the woman was not pregnant was extrapolated for the whole year. Miscarriages and terminations of pregnancy (TOP) were recorded separately.

\section{Criteria for recording consultation}

All general practice consultations with the doctor which were documented in the medical records were counted for the year prior to conception and the year after birth. Consultations with practice nurses for illness or contraception were included. However, consultations solely for blood taking, wound dressing, and immunisations were excluded as practice nurse provision of these services changed considerably during the study. Saturday morning surgeries and daytime visits were included but out of hours visits and telephone advice were excluded, due to unreliable recording. GP-initiated visits at the time of delivery for routine new-born examination were not included, but woman- or midwife-requested postnatal visits and the 6 week maternal postnatal examination were included.

Where inspection of the mother's medical records found any mention in the year being examined of infertility, life events and symptoms of anxiety or depression, this information was extracted. These were considered clinically important influences on consulting behaviour. Anxiety and depression scales were not routinely used by the GPs. When events (e.g., bereavement, relationship break-up, marriage) were mentioned in the notes which met the criteria for 'life events' as defined by Kumar and Robson (Kumar and Robson, 1984), they were counted but pregnancy events were excluded.

The baby consultation rate did not include visits to the child health clinic as significant changes in immunisation and developental assessments occurred during the study and only 191 health visitor records were available.

\section{Socio-economic data recording}

Postcodes and occupational data were taken from medical records or if these were missing, from the maternity record. Occupational class was that of the father of the baby for couples and the mother's occupation for single mothers. It was classified according to current definitions used by The Office of Population Censuses and Surveys Census. Two categories were used, nonmanual (class I-IIIn) and manual (class IIIm-V)/unemployed/students. 


\section{Statistical methods}

Univariate comparisons between the place of birth (home, domino and hospital) were performed using the Chi-squared test for categorical variables (e.g., percentage primiparous); one way analysis of variance for continuous normally distributed variables (e.g., baby birth weight, maternal age); and the Kruskal-Wallis test for continuous nonnormally distributed variables (e.g., combined mother and baby GP consultation rate for the year following birth). To investigate the independent variables (e.g., actual place of birth, occupational class) associated with GP consultation rates (log transformed) multiple linear regression was performed. The combined mother and baby consultation rates in the year after birth (CMBFB), the difference between the combined consultation rate for the year following birth minus the year preceding conception (DCMBFB) and the number of postnatal mother and baby consultations as a percentage of the total mother and baby consultations (PCMBFB) were the outcome variables of interest. The pre-birth variables entered into the regression analysis with intended place of birth were: maternal age, parity, smoking during pregnancy; occupational class; number of miscarriages and any recorded history of infertility, depression or anxiety, life events in year preceding conception. The independent variables entered into the regression analysis with actual place of birth were the above variables and the additional postnatal variables: birth weight, sex of baby, breastfeeding initiation, breastfeeding at 6 weeks; any recorded depression or anxiety in year following birth and life events in year following birth. Any $P$ value $<0.05$ was considered statistically significant. All statistical analyses were performed using SPSS for Windows, version 9 (SPSS Inc, Chicago, IL, USA).

\section{Results}

\section{Characteristics of mothers included and excluded from the study}

The mothers included in the study were older $(P<0.001)$, less likely to be primiparous $(P<0.001)$ and more likely to have a home birth $(P<0.001)$ than those excluded. They did not differ in ethnicity (Table 1).
Pre-pregnancy, pregnancy and postnatal characteristics of the study sample (Table 2)

Some mothers in the study had more than one pregnancy included: 243 women had just one pregnancy included, 78 women had two pregnancies, 14 women had three pregnancies and three women had four pregnancies. Mothers with anxiety or depression recorded in their notes increased from $45 / 453(9.9 \%)$ in the year preceding conception to $71 / 453(15.7 \%)$ in the year after birth $(P=0.002)$. There was no similar increase in the number of life events recorded. Fifty-nine (13\%) miscarriages/ TOP were observed pre-conception from 56 women. Nine women had a miscarriage/TOP in the year post-birth. Thirty-eight women gave birth to another child in the pre-conception year and 34 women were pregnant within a year of the birth. Postnatal examinations were performed by the GP

Table 2 Antenatal, intra-partum and postnatal characteristics of the 453 births included in the study

\begin{tabular}{|c|c|}
\hline \multicolumn{2}{|l|}{$\begin{array}{l}\text { Pre-pregnancy (year prior to } \\
\text { conception) }\end{array}$} \\
\hline Infertility $n(\%)$ & $29(6.4)$ \\
\hline Depression or anxiety $n(\%)$ & $45(9.9)$ \\
\hline Life events $n(\%)$ & $35(7.7)$ \\
\hline Number of miscarriages/TOP $n(\%)$ & $59(13.0)$ \\
\hline \multicolumn{2}{|l|}{ During-pregnancy } \\
\hline $\begin{array}{l}\text { Smoked between conception } \\
\text { and } 1 \text { year }\end{array}$ & $131(28.9)$ \\
\hline \multicolumn{2}{|l|}{ At birth } \\
\hline Baby sex, male $n(\%)$ & $240(53 \%)$ \\
\hline Mean gestational age (SD) (range) & $\begin{array}{l}40.1(1.5)[37- \\
45]\end{array}$ \\
\hline Mean birth weight (g) (SD) (range) & $\begin{array}{l}3406(472) \\
{[1750-4900]}\end{array}$ \\
\hline \multicolumn{2}{|l|}{ Post-pregnancy ( 1 year after birth) } \\
\hline Depression or anxiety $n(\%)$ & $71(15.7)$ \\
\hline Life events $n(\%)$ & $37(8.2)$ \\
\hline Number of miscarriages/TOP $n(\%)$ & $9(2.0)$ \\
\hline \multicolumn{2}{|l|}{ Immediately after birth $n(\%)$} \\
\hline $\begin{array}{l}\text { Breastfeeding } \\
\text { Bottle }\end{array}$ & $\begin{array}{l}276(60.9) \\
174(38.4)\end{array}$ \\
\hline Unknown & $3(0.7)$ \\
\hline \multicolumn{2}{|l|}{ Six weeks after birth $n(\%)$} \\
\hline Breastfeeding & $212(46.8)$ \\
\hline Bottle & $236(52.1)$ \\
\hline Unknown & $5(1.1)$ \\
\hline \multicolumn{2}{|l|}{$\begin{array}{l}\text { Number of pregnancies per woman } \\
\text { included in the study } n(\%)\end{array}$} \\
\hline One pregnancy & $243(53.6)$ \\
\hline Two pregnancies & $78(17.2)$ \\
\hline Three pregnancies & $14(3.1)$ \\
\hline Four pregnancies & $3(0.7)$ \\
\hline
\end{tabular}


for 435 women, six were done at hospital and 12 mothers did not have one recorded.

\section{Relationship between intended place of birth and actual place of birth}

Of the 86 mothers who planned a home birth, 64 successfully delivered at home and six mothers changed to the domino scheme, four antenatally and two during labour (Table 3). Sixteen mothers were transferred to hospital care, 10 antenatally and six during labour. One baby was born in the ambulance and in subsequent analyses was included with the hospital births. Of the 126 mothers who intended a domino birth, 89 actually had a domino birth. Four women requested to change to a home birth prior to delivery, one other had an unplanned home birth, while the remaining 32 were transferred to hospital care. Of the 240 mothers who planned a hospital birth, 238 mothers had a hospital birth and two requested antenatally to change to a domino birth. One woman had a concealed pregnancy and was not booked for maternity care. She is therefore not included in the intended place of birth analyses $(n=452)$, but is included for actual place of delivery analyses $(n=453)$. The reasons for transfer from intended place of delivery to actual place of delivery are set out in Table 4.

\section{The influence of intended and actual place of birth on mother and baby consultation rates}

The central analysis was to look at intended and actual place of birth on mother and baby consultation patterns, adjusting for key sociodemographic (i.e., maternal age and occupational class) and clinical variables (parity, infertility, anxiety or depression, life events, smoking during pregnancy, number of miscarriages in year preced- ing conception, breastfeeding, sex of baby and birth weight) (Table 5).

Mothers intending a home birth were older $(P<0.001)$, less likely to be primiparous $(P=$ $0.055)$ and less likely to be of manual occupational class $(P<0.001)$ (Table 6). Additional associations for mothers actually having a home birth were heavier babies $(P<0.001)$, less perineal trauma $(P<0.001)$ and higher breastfeeding initiation at birth $(P=0.001)$ and at 6 weeks $(P<0.001)$ (Table 7$)$. The mean weight of babies born to primiparous women was less (mean $3353 \mathrm{~g}$, SD $449 \mathrm{~g}$ ) than the mean weight of babies born to multiparous women (mean $3436 \mathrm{~g}$, SD $483 \mathrm{~g})$, but this was not significant $(P=0.07)$. Women who smoked during pregnancy had significantly smaller babies compared with nonsmokers (mean (SD): $3330 \mathrm{~g}$ (449 g) versus $3436 \mathrm{~g}$ (479 g) respectively, $P=0.03$ ).

After adjustment for maternal age, occupational class, parity, infertility, life events pre-birth and anxiety or depression pre-birth, smoking during pregnancy and miscarriages in the year preceding conception, intended place of birth was found to be independently associated with mother and baby consultation rates following birth (CMBFB). Mothers intending a home birth had a lower CMBFB rate than mothers intending domino births $(P=0.002)$ and mothers intending hospital births $(P<0.001)$ (Table 6). Mothers who had higher CMBFB rates were significantly more likely to be primiparous $(P=0.001)$, of manual occupational class $(P=0.012)$ or smokers $(P<0.001)$. In addition, prior to pregnancy, they were more likely to have experienced infertility problems $(P=$ $0.025)$, anxiety or depression $(P<0.001)$, or report a life event $(P=0.008)$. All these variables were independently associated with increased con-

Table 3 Intended and actual place of birth

Intended

Actual

Home Domino Hospital Ambulance Total

\begin{tabular}{lrrrrr}
\hline Home & 64 & 6 & 15 & 1 & 86 \\
Domino & 5 & 89 & 32 & 0 & 126 \\
Hospital & 0 & 2 & 238 & 0 & 240 \\
Not booked & 0 & 0 & 1 & 0 & 1 \\
Total & 69 & 97 & 286 & 1 & 453 \\
\hline
\end{tabular}


Table 4 Reasons for transfer from intended place of birth to actual place of birth

\begin{tabular}{|c|c|c|c|}
\hline Reason & $\begin{array}{l}\text { Transfer from } \\
\text { intended home } \\
\text { birth to domino } \\
\text { birth } n=6\end{array}$ & $\begin{array}{l}\text { Transfer from } \\
\text { intended home } \\
\text { birth to hospital } \\
\text { care birth } n=16\end{array}$ & $\begin{array}{l}\text { Transfer from } \\
\text { intended domino } \\
\text { birth to hospital } \\
\text { care birth } n=32\end{array}$ \\
\hline
\end{tabular}

\section{Antenatal transfer}

Low placenta

Impaired glucose tolerance

APH

Gestational diabetes

Breech

Mitral valve disease

Maternal thrombocytopenia

Rising Rhesus antibody titre

Anti C maternal antibodies

Suspected intra-uterine growth retardation

Pregnancy induced hypertension

Suspected fetal distress

Unexplained right iliac fossa pain

\section{Transfer in labour}

\section{$\mathrm{APH}$}

Delay 1 st stage of labour

Delay 2 nd stage of labour

Suspected fetal distress

Fetal distress 1st stage of labour

Fetal distress 2nd stage of labour

Prolonged spontaneous rupture of membranes

Born at another hospital while staying away from home

Reason not recorded
1

1

$\begin{array}{ll}2 & 1 \\ 1 & 1 \\ 3 & 2 \\ 1 & \\ 1 & \\ & \\ 1 & 1 \\ & 3 \\ & 1 \\ & 1\end{array}$

1

1

1

1 sultations. Interestingly, ethnicity was not found to be independently associated with increased consultations.

When pre-existing ill health was accounted for by subtracting the number of consultations made by the mother in the year prior to conception from the combined mother and baby consultations in the year after birth (DCMBFB), mothers intending a home birth still had a lower consultation rate than mothers intending a domino birth or hospital birth $(P<0.001)$ (Table 6).

Actual place of birth was also independently associated with mother and baby consultation rates following birth. Home births were observed to have fewer CMBFB compared to domino births $(P=0.015)$, hospital births $(P=0.001)$ and assisted hospital births $(P=0.007)$. Other maternal characteristics which were independently associated with CMBFB were: primiparity $(P=0.02)$, not breastfeeding initially $(P<0.001)$, maternal smoking $(P=0.004)$, infertility problems prior to pregnancy $(P=0.014)$, life events $(P=0.004)$ and anxiety or depression noted post-birth $(P<0.001)$. Breastfeeding at 6 weeks, life-events pre-birth and anxiety or depression pre-birth were also independent variables associated with consultation rates, but could not be included in the same regression model as breastfeeding initiation, life events post-birth and anxiety or depression postbirth respectively due to co-linearity (e.g., most of the mothers who breastfed at initiation also breastfed 6 weeks later, these two variables are highly correlated and cannot be included in the same regression model). The distribution of CMBFB rate is presented for all the maternal characteristics that are independently associated with CMBFB (see Table 8). When pre-existing ill health was accounted for (DCMBFB), mothers having a home birth still had a lower consultation rate than mothers having a domino birth or hospital birth $(P<0.001)$ (Table 7$)$.

Parity was the only independent predictor of the 
Table 5 The key socio-demographic and clinical variables adjusted for in the analysis of the influence of intended and actual place of birth on mother and baby consultation rates

Variables adjusted for in the analysis of the relationship between intended place of birth and mother and baby consultation rates
Variables adjusted for in the analysis of the relationship between actual place of birth and mother and baby consultation rates
Maternal age

Occupational class

Parity

Infertility recorded in the year prior to conception

Anxiety or depression recorded in the year prior to conception

Life events recorded in the year prior to conception

Smoking during pregnancy

Number of miscarriages/terminations of pregnancy in year prior to conception

\author{
Maternal age \\ Occupational class \\ Parity \\ Infertility recorded in the year prior to conception \\ Anxiety or depression recorded in the year prior to \\ conception \\ Anxiety or depression recorded in the first postnatal \\ year \\ Life events recorded in the year prior to conception \\ Life events recorded in the first postnatal year \\ Smoking during pregnancy \\ Number of miscarriages/terminations of pregnancy in \\ year prior to conception \\ Number of miscarriages/terminations of pregnancy in \\ the first postnatal year \\ Birth weight \\ Sex of baby \\ Breastfeeding initiation after birth \\ Breastfeeding at 6 weeks
}

percentage of total mother and baby consultations pre- and post-birth which occurred in the first postnatal year (PCMBFB). Primiparous mothers were observed to have a higher percentage of their total consultations in the year following birth compared with multiparous mothers $(P<0.001)$.

For all of the above analyses, the unit of analysis has been pregnancy and it has been assumed that the pregnancies are independent. However in this sample, 95 of the 338 mothers had more than one pregnancy included in the study. Therefore all the data have been re-analysed (Tables 9 and 10) including only the first pregnancy of each woman and the results compared with the whole cohort. None of the findings were different to the above results from the 453 pregnancies from 338 mothers. Obviously more of these pregnancies were primiparous, but still the same independent associations with consultation rates and place of delivery were observed.

\section{Discussion}

This study provides important new information about how consultation patterns for mothers and babies in the year following childbirth differ according to place of birth. The key finding is that there is an association between women intending and actually having home births and lower mother and baby consultation rates postnatally compared with women having domino deliveries with the same known professional team caring for them in labour and women having normal vaginal deliveries in hospital with a different professional team caring for them. This effect persists when preexisting maternal ill health is accounted for. The effect of intended and actual place of birth is independent of maternal age, parity, smoking during pregnancy, occupational class, a history of infertility or miscarriage in the year prior to conception, anxiety, depression or life events in the year prior to conception or after birth, breastfeeding, sex of baby and birth weight.

\section{Comparison with other studies}

The actual home birth rate of $15 \%(69 / 453)$ women included in this study and the overall actual home birth rate of $9.3 \%(95 / 1021)$ for the practice during the 10 year study period are higher than the national average of $2.2 \%$ in 1998 , but other characteristics of the sample are comparable with 
Table 6 Maternal characteristics and consultation rates according to intended place of birth (one birth not booked)

$\begin{array}{lll}\begin{array}{l}\text { Home birth } \\ (n=86)\end{array} & \begin{array}{l}\text { Domino birth } \\ (n=126)\end{array} & \begin{array}{l}\text { Hospital birth } \\ (n=240)\end{array}\end{array} \quad P$ value

Maternal characteristics
Mean maternal age (SD)
Primiparous $n(\%)$
Maternal smoking $n(\%)$
Occupational class, manual $n(\%)$
History of infertility $n(\%)$
Depression or anxiety pre-pregnancy $n(\%)$
Life events pre-pregnancy $n(\%)$
Number of miscarriages/TOPs
pre-pregnancy $n(\%)$

$\begin{array}{cc}31.6(4.5) & 29.3(5.3) \\ 22(25.6) & 44(34.9) \\ 19(22.6) & 35(27.8) \\ 30(35.7) & 53(42.1) \\ 5(5.8) & 7(5.6) \\ 7(8.1) & 10(7.9) \\ 5(5.8) & 7(5.6) \\ 13(15.1) & 16(12.7)\end{array}$

Consultation rates

Median CMBFB (range)

Median DCMBFM (range)

Median PCMBFB (range)

$\begin{array}{lc}8(1,56.2) & 10.5(1,40.4) \\ 4.4(-8.2,43.2) & 7(-4,29) \\ 75(33-100) & 74.6(16.7-100)\end{array}$

$\begin{array}{rr}28.4(5.4) & <0.001^{\mathrm{a}} \\ 96(40) & 0.055^{\mathrm{b}} \\ 76(31.7) & 0.275^{\mathrm{b}} \\ 169(71.3) & <0.001^{\mathrm{b}} \\ 17(7.1) & 0.825^{\mathrm{b}} \\ 28(11.7) & 0.433^{\mathrm{b}} \\ 23(9.6) & 0.297^{\mathrm{b}} \\ 30(12.5) & 0.818^{\mathrm{b}}\end{array}$

CMBFB, combined mother and baby GP consultation rate in year following birth; DCMBFB, combined mother and baby GP consultation rate in year following birth minus the mothers' consultation rate in the year preceding conception; PCMBFB, the number of postnatal mother and baby consultations as a percentage of the total mother and baby consultations:

$\mathrm{PCMBFB}=\frac{\mathrm{CMBFB}}{\text { (mothers consultations pre-conception }+ \text { CMBFB })} \times 100$

${ }^{a}$ One way analysis of variance.

${ }^{\mathrm{b}}$ Chi-squared test.

${ }^{\mathrm{c}}$ Kruskal-Wallis test.

national data (Foster et al., 1997; Macfarlane and Mugford, 2000). Our data show similar associations between lower birth weight and both smoking and primiparity. The home birth rate may be higher than the national average for several reasons. The predominantly female doctors in the practice had a local reputation with both patients and midwives for promoting choice in childbirth which may have attracted women wanting a home birth to register with the practice. Written and verbal information about the availability of home births was given to all pregnant women. Positive experiences of home births were discussed in the community, at childbirth groups (e.g., The National Childbirth Trust) and awareness of this as an option increased. The midwives were confident in their home delivery skills and did not discourage it unless there was a contraindication.

The fourth national morbidity survey in general practice in 1991/1992 records a consultation rate for all general practice contacts of 4.6 per 10000 person years at risk for women aged 16-44 years and a rate of 5.5 and 5.3 for male and female children aged 0-4 years, respectively (Royal College of General Practitioners, Office of Population Censuses and Surveys, and Department of Health, 1995). It is not possible to directly compare these with our combined mother and baby consultation rates of seven per year for women having a home birth and 12 per year for women having a normal hospital delivery. The finding that there is an association between mother-infant pairs who breastfed and lower consultation rates compared with formula-fed pairs supports the existing evidence that breastfeeding reduces childhood morbidity in the first year of life (Howie et al., 1990). However, there were associations between mothers who had a home birth and breastfeeding, and between mothers who had a home birth and lower reported levels of anxiety and smoking during pregnancy, which could also contribute to fewer consultations. 
Table 7 Maternal characteristics and consultation rates according to actual place and method of birth

\begin{tabular}{|c|c|c|c|c|c|}
\hline Maternal and birth characteristics & $\begin{array}{l}\text { SVD home birth } \\
(n=69)\end{array}$ & $\begin{array}{l}\text { SVD domino } \\
\text { birth }(n=97)\end{array}$ & $\begin{array}{l}\text { SVD hospital } \\
\text { birth }(N=201)\end{array}$ & $\begin{array}{l}\text { Assisted hospital } \\
\text { birth }(n=86)\end{array}$ & $P$ value \\
\hline Mean maternal age (SD) & $31.2(4.6)$ & $29.2(5.5)$ & $28.5(5.4)$ & $29.4(5.2)$ & $0.003^{\mathrm{a}}$ \\
\hline Primiparous $n(\%)$ & $10(14.5)$ & $32(33.0)$ & $69(34.3)$ & $52(60.5)$ & $<0.001^{b}$ \\
\hline Maternal smoking $n(\%)$ & $13(19.4)$ & $30(30.9)$ & $64(31.8)$ & $24(27.9)$ & $0.261^{b}$ \\
\hline Occupational class, manual $n(\%)$ & $24(35.8)$ & $42(43.3)$ & $140(70.4)$ & $47(55.3)$ & $<0.001^{b}$ \\
\hline History of infertility $n(\%)$ & $3(4.3)$ & $6(6.2)$ & $13(6.5)$ & $7(8.1)$ & $0.819^{\mathrm{b}}$ \\
\hline $\begin{array}{l}\text { Depression or anxiety pre-pregnancy } \\
n(\%)\end{array}$ & $5(7.2)$ & $8(8.2)$ & $22(10.9)$ & $10(11.6)$ & $0.712^{b}$ \\
\hline $\begin{array}{l}\text { Depression or anxiety post- } \\
\text { pregnancy } n(\%)\end{array}$ & $8(11.6)$ & $11(11.3)$ & $35(17.4)$ & $17(19.8)$ & $0.284^{b}$ \\
\hline Life events pre-pregnancy $n(\%)$ & $4(5.8)$ & $5(5.2)$ & $17(8.5)$ & $9(10.5)$ & $0.510^{\mathrm{b}}$ \\
\hline Life events post-pregnancy $n(\%)$ & $5(7.2)$ & $5(5.2)$ & $19(9.5)$ & $8(9.3)$ & $0.606^{b}$ \\
\hline $\begin{array}{l}\text { Number of miscarriages/TOP pre- } \\
\text { pregnancy } n(\%)\end{array}$ & $7(10.1)$ & $12(12.4)$ & $26(12.9)$ & $14(16.3)$ & $0.718^{b}$ \\
\hline $\begin{array}{l}\text { Number of miscarriages/TOP post- } \\
\text { pregnancy } n(\%)\end{array}$ & $3(4.3)$ & $1(1.0)$ & $4(2.0)$ & $1(1.2)$ & \\
\hline Breastfeeding at birth $n(\%)$ & $56(82.4)$ & $57(58.8)$ & $119(59.5)$ & $44(51.8)$ & $0.001^{\mathrm{b}}$ \\
\hline Breastfeeding at 6 weeks $n(\%)$ & 53 (77.9) & $46(48.4)$ & $82(41.0)$ & 31 (36.5) & $<0.001^{b}$ \\
\hline Sex of baby, male $n(\%)$ & $34(49.3)$ & $55(56.7)$ & $108(53.7)$ & $43(50)$ & $0.736^{b}$ \\
\hline Mean birth weight (g) (SD) & $3618(489)$ & $3380(434)$ & 3338 (457) & 3427 (493) & $<0.001^{\mathrm{a}}$ \\
\hline \multicolumn{6}{|l|}{ Perineal trauma $n(\%)$} \\
\hline Intact & $39(62.9)$ & $48(50.0)$ & $107(54.0)$ & $45(54.2)$ & $<0.001^{b}$ \\
\hline 1st degree tear & $9(14.5)$ & $17(17.7)$ & $25(12.6)$ & $1(1.2)$ & \\
\hline 2nd degree tear & $14(22.6)$ & $26(27.1)$ & $46(23.2)$ & $6(7.2)$ & \\
\hline 3rd degree tear & 0 & 0 & 0 & $1(1.2)$ & \\
\hline Episiotomy & 0 & $5(5.2)$ & $20(10.1)$ & $30(36.1)$ & \\
\hline \multicolumn{6}{|l|}{ Consultation rates } \\
\hline Median CMBFB (range) & $7(1,56.2)$ & $10(3,40.4)$ & $12(1,39)$ & $11(0,52)$ & $<0.001^{c}$ \\
\hline Median DCMBFM (range) & $\begin{array}{l}4.4(-8.2 \\
43.2)\end{array}$ & $7(-2,29)$ & $7(-5,29)$ & $8.8(-3,33)$ & $<0.001^{c}$ \\
\hline Median PCMBFB (range) & $70(33.3-100)$ & $74.3(37.5-100)$ & $73.3(16.7-100)$ & $77.8(0-100)$ & $0.058^{c}$ \\
\hline
\end{tabular}

Assisted hospital births includes forceps, ventouse and caesarian births.

CMBFB, combined mother and baby GP consultation rate in year following birth; DCMBFB, combined mother and baby GP consultation rate in year following birth minus the mothers' consultation rate in the year preceding conception; PCMBFB, the number of postnatal mother and baby consultations as a percentage of the total mother and baby consultations:

PCMBFB $=\frac{\text { CMBFB }}{\text { (mothers consultations pre-conception }+ \text { CMBFB })} \times 100$

${ }^{a}$ One way analysis of variance.

${ }^{\mathrm{b}} \mathrm{Chi}$-squared test.

${ }^{c}$ Kruskal-Wallis test.

\section{Study design issues}

A strength of this study is the consistency of primary care and maternity care provision and data recording. This reduces the likelihood that the observed differences in consultation rates for home births are due to practice factors. However, the generalisability of this data is unknown, as it is one practice with a heterogeneous population and consultation behaviour can be culturally deter- mined (Royal College of General Practitioners, Office of Population Censuses and Surveys, and Department of Health 1995; Saxena et al., 1999). There are other important limitations with the study design. Firstly, although we adjusted for some maternal and infant characteristics in the analysis, there is likely to be residual confounding factors which could be operating at both the choice of intended place of birth and the medical reason 
Table 8 Distribution of the combined mother and baby GP consultation rate in year following birth (CMBFB) for the maternal characteristics independently associated with CMBFB

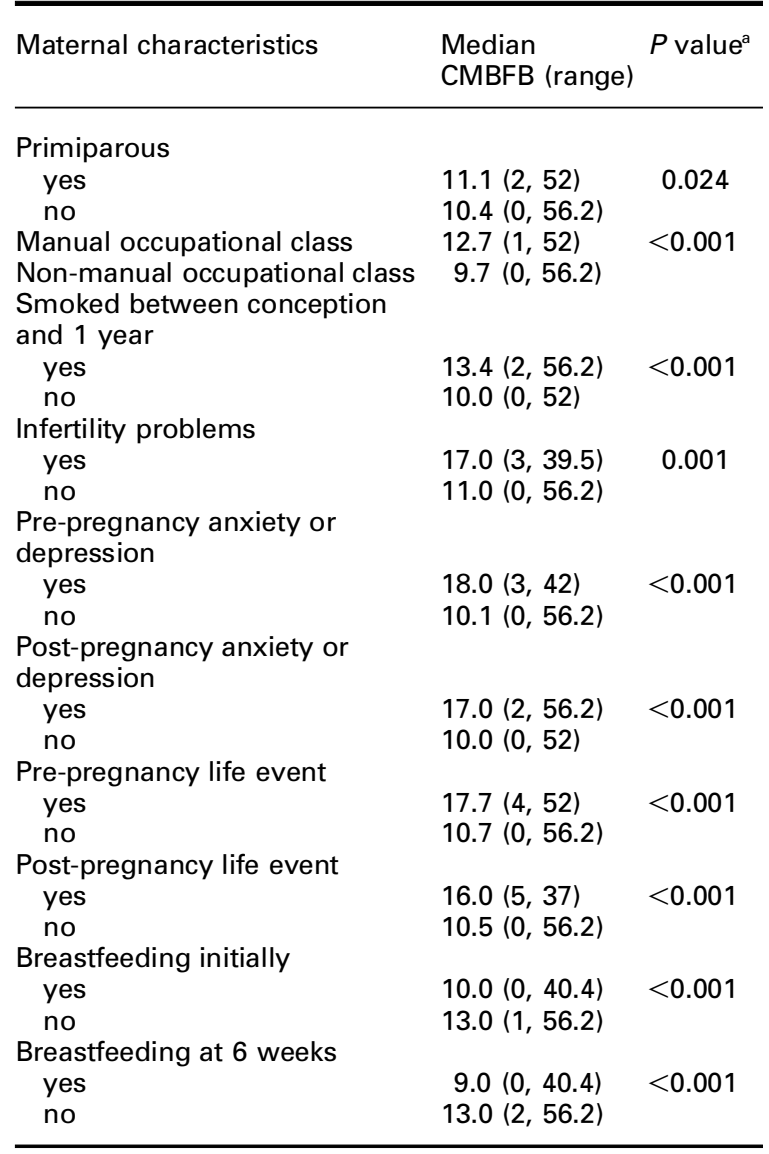

${ }^{a}$ Mann-Whitney U-test.

for actual place of birth and provision of care, for example health beliefs or previous experience of illness. Secondly, while the maternity-related data were collected prospectively with the aim of looking at outcomes of place of birth, the consultation data were collected retrospectively. Such data necessarily rely on the consistency and the completeness of information recorded at the time and it is also possible that some consultations were not recorded, further reducing the reliability of the data. The retrospective consultation data collection combined with the high patient mobility resulted in only $44 \%$ of births during the study period being included, with a bias towards older and multip- arous mothers. Mobile women are likely to be younger and in less stable relationships. The inclusion of a higher proportion of home births is due to $\mathrm{PH}$ photocopying the notes of those women who gave birth at home when she knew they were leaving the practice. This could contribute a bias if women leaving the practice differed in their consultation behaviour. Thirdly, the exclusion of data on out-of-hours consultations and child health clinic consultations is also a limitation. We do not know whether place of birth is associated with attendance at health visitor clinics and this would be important to include in future studies. By including women who became pregnant either in the year pre-conception or post-birth and extrapolating the consultation rate, we may have overestimated the postnatal consultation rate, as the mother's consultation rate included the postnatal examination at 6 weeks. However, no socio-demographic differences (parity, smoking, occupational class and ethnicity) were observed between the women who had a pregnancy in the year after birth $(n=91)$ and women who did not $(n=247)$.

The consultation data on life events, depression, anxiety, smoking and infertility data were dependent on the GP recording at the time of consultation and therefore may be under-recorded. In particular, GPs were not using standardised tools to diagnose life events, depression or anxiety and definitions may be inconsistent both within an individual doctor over time and between doctors. However, the $15.7 \%$ prevalence of postnatal anxiety and depression is consistent with the nationally reported prevalence of post-natal depression of 1015\% (O'Hara and Swain, 1996). The weaknesses of using occupational class when looking at morbidity data have been documented (Haynes, 1991).

This study took place at a time when the role of the GP in maternity care was changing both locally and nationally, with many GPs withdrawing from intra-partum care. Ideally, the role of the GP would have remained constant throughout the study period to avoid the potential bias of the GPs involvement at birth influencing consultation rates. However, we do not think this change in role had any influence on the study outcome.

Despite these limitations, this study is the first to document variations in GP consultation rates analysed by intended and actual place of birth. It is also original in taking into account maternal consultations prior to conception in an attempt to mea- 
Table 9 Maternal characteristics and consultation rates according to intended place of birth (one birth not booked) for the first recorded pregnancy of each woman

$$
\begin{aligned}
& \text { Home birth }(n=47) \text { Domino birth Hospital birth } P \text { value } \\
& (n=96) \quad(n=194)
\end{aligned}
$$

\begin{tabular}{lccrr} 
Maternal characteristics & & & \\
Mean maternal age (SD) & $31.5(4.3)$ & $29.1(5.4)$ & $27.8(5.3)$ & $<0.001^{\mathrm{a}}$ \\
Primiparous $n(\%)$ & $22(46.8)$ & $44(45.8)$ & $96(49.5)$ & $0.828^{\mathrm{b}}$ \\
Maternal smoking $n(\%)$ & $7(15.6)$ & $30(31.3)$ & $63(32.5)$ & $0.077^{\mathrm{b}}$ \\
Occupational class, manual $n(\%)$ & $15(32.6)$ & $42(43.8)$ & $133(69.3)$ & $<0.001^{\mathrm{b}}$ \\
History of infertility $n(\%)$ & $2(4.3)$ & $7(7.3)$ & $15(7.7)$ & $0.706^{\mathrm{b}}$ \\
Depression or anxiety pre-pregnancy $n(\%)$ & $5(10.6)$ & $6(6.3)$ & $19(9.8)$ & $0.549^{\mathrm{b}}$ \\
Life events pre-pregnancy $n(\%)$ & $5(10.6)$ & $7(7.3)$ & $18(9.3)$ & $0.773^{\mathrm{b}}$ \\
Number of miscarriages/TOPs pre-pregnancy $n(\%)$ & $7(14.9)$ & $13(13.5)$ & $25(12.9)$ & $0.934^{\mathrm{b}}$ \\
Consultation rates & & & & \\
Median CMBFB (range) & & & \\
Median DCMBFB (range) & $8(1,56.2)$ & $10.7(1,39.5)$ & $12.4(0,52)$ & $<0.001^{\mathrm{c}}$ \\
Median PCMBFB (range) & $4(-2,43.2)$ & $7.2(-4,29)$ & $8(-5,33)$ & $0.001^{\mathrm{c}}$ \\
\hline
\end{tabular}

CMBFB, combined mother and baby GP consultation rate in year following birth; DCMBFB, combined mother and baby GP consultation rate in year following birth minus the mothers' consultation rate in the year preceding conception; PCMBFB, the number of postnatal mother and baby consultations as a percentage of the total mother and baby consultations:

PCMBFB $=\frac{\text { CMBFB }}{\text { (mothers consultations pre-conception }+ \text { CMBFB })} \times 100$

${ }^{a}$ One way analysis of variance.

${ }^{\mathrm{b}}$ Chi-squared test.

${ }^{\mathrm{c}}$ Kruskal-Wallis test.

sure the increase in consultations attributable to childbirth. The percentages of total consultations pre- and post-birth for mother and baby which occur postnatally are very similar for all birth intention groups, but differ for actual place of birth. There is a strong trend, which does not quite reach significance at the $5 \%$ level, showing a gradient with home births associated with the lowest percentage of postnatal consultations, similar rates for domino and normal vaginal hospital births and assisted hospital births having the highest. This suggests that where and how a baby is born may be associated with postnatal consultation patterns in general practice. Although women intending a home birth might be expected to behave more independently of medical services, as far as we are aware there is no published data in this field. If our findings are confirmed and the trend in maternity care continues to move away from normal delivery and home births towards caesarian section, workload in general practice in the first post-natal year could increase considerably.

Of course, it is not possible to determine the extent to which the increase in consultation rate after childbirth reflects true mother and baby morbidity, nor draw conclusions about why these important differences in consultation rates occur. Further research is needed to determine the relative importance of characteristics like maternal confidence, health beliefs, self-reliance and attitudes to consulting health professionals which are likely to influence both place of birth and general practice consultation patterns.

\section{Acknowledgements}

We would like to thank GPs and staff at St. Stephen's Health Centre, London E3 5ED and in particular Lorraine Chapman for maintaining the maternity register throughout the study. We also thank Nigel Stott, Ian Russell and Charis Glazener for their helpful comments. PH received funding from Grampian Healthcare NHS Trust and Grampian Primary Care NHS Trust to write up this research. Macduff Medical Practice is a Scottish Office Department of Health Research Practice. 
Table 10 Maternal characteristics and consultation rates according to actual place and method of birth for the first recorded pregnancy of each woman

\begin{tabular}{|c|c|c|c|c|c|}
\hline Maternal and birth characteristics & $\begin{array}{l}\text { SVD home birth } \\
(n=36)\end{array}$ & $\begin{array}{l}\text { SVD domino } \\
\text { birth }(n=69)\end{array}$ & $\begin{array}{l}\text { SVD hospital } \\
\text { birth }(n=159)\end{array}$ & $\begin{array}{l}\text { Assisted hospital } \\
\text { birth }(n=74)\end{array}$ & $P$ value \\
\hline Mean maternal age (SD) & $31.3(4.3)$ & $29.0(5.6)$ & $27.8(5.3)$ & $29.0(5.4)$ & $0.003^{a}$ \\
\hline Primiparous $n(\%)$ & $10(27.8)$ & $32(46.4)$ & $69(43.4)$ & $52(70.3)$ & $<0.001^{b}$ \\
\hline Maternal smoking $n(\%)$ & $5(14.7)$ & $24(34.8)$ & $50(31.4)$ & $22(29.7)$ & $0.196^{b}$ \\
\hline Occupational class, manual $n(\%)$ & $11(31.4)$ & $32(46.4)$ & $108(68.8)$ & $40(54.1)$ & $<0.001^{\mathrm{b}}$ \\
\hline History of infertility $n(\%)$ & $1(2.8)$ & $6(8.7)$ & $11(6.9)$ & $6(8.1)$ & $0.704^{\mathrm{b}}$ \\
\hline $\begin{array}{l}\text { Depression or anxiety } \\
\text { pre-pregnancy } n(\%)\end{array}$ & $4(11.1)$ & $5(7.2)$ & $15(9.4)$ & $6(8.1)$ & $0.905^{\mathrm{b}}$ \\
\hline $\begin{array}{l}\text { Depression or anxiety } \\
\text { post-pregnancy } n(\%)\end{array}$ & $4(11.1)$ & $7(10.1)$ & $26(16.4)$ & $13(17.6)$ & $0.507^{\mathrm{b}}$ \\
\hline Life events pre-pregnancy $n(\%)$ & $4(11.1)$ & $5(7.2)$ & $12(7.5)$ & $9(12.2)$ & $0.618^{b}$ \\
\hline Life events post-pregnancy $n(\%)$ & $4(11.1)$ & $4(5.8)$ & $15(9.4)$ & 7 (9.5) & $0.771^{\mathrm{b}}$ \\
\hline $\begin{array}{l}\text { Number of miscarriages/TOP pre- } \\
\text { pregnancy } n(\%)\end{array}$ & $4(11.1)$ & $10(14.5)$ & $19(11.9)$ & $12(16.2)$ & $0.794^{\mathrm{b}}$ \\
\hline $\begin{array}{l}\text { Number of miscarriages/TOP } \\
\text { post-pregnancy } n(\%)\end{array}$ & $3(8.3)$ & $1(1.4)$ & $4(2.5)$ & $1(1.4)$ & \\
\hline Breastfeeding at birth $n(\%)$ & $30(83.3)$ & $40(58.0)$ & $94(59.5)$ & $37(50.7)$ & $0.012^{\mathrm{b}}$ \\
\hline Breastfeeding at 6 weeks $n(\%)$ & $29(80.6)$ & 33 (49.3) & 63 (39.9) & $24(32.9)$ & $<0.001^{b}$ \\
\hline Sex of baby, male $n(\%)$ & $13(36.1)$ & $39(56.5)$ & $86(54.1)$ & $36(48.6)$ & $0.189^{b}$ \\
\hline Mean birth weight (g) (SD) & 3569 (458) & 3361 (458) & 3296 (433) & $3408(490)$ & $0.010^{\mathrm{a}}$ \\
\hline \multicolumn{6}{|l|}{ Perineal trauma $n(\%)$} \\
\hline Intact & $19(63.3)$ & $31(45.6)$ & $85(53.8)$ & $38(53.5)$ & $<0.001^{b}$ \\
\hline 1st degree tear & $5(16.7)$ & $13(19.1)$ & $16(10.1)$ & $1(1.4)$ & \\
\hline 2nd degree tear & $6(20.0)$ & $21(30.9)$ & $38(24.1)$ & $6(8.5)$ & \\
\hline 3rd degree tear & 0 & 0 & 0 & $1(1.4)$ & \\
\hline Episiotomy & 0 & $3(4.4)$ & $19(12.0)$ & $25(35.2)$ & \\
\hline \multicolumn{6}{|l|}{ Consultation rates } \\
\hline Median CMBFB (range) & $7(1,56.2)$ & $10(3,36)$ & $12(1,35)$ & $11(0,52)$ & $0.003^{c}$ \\
\hline Median DCMBFB (range) & $3.5(-2,43.2)$ & $7(-2,29)$ & $7(-5,29)$ & $9(-2,33)$ & $0.001^{c}$ \\
\hline Median PCMBFB (range) & $68.3(33.3,100)$ & $75(37.5,100)$ & $75(16.7,100)$ & $78(0,100)$ & $0.055^{c}$ \\
\hline
\end{tabular}

Assisted hospital births includes forceps, ventouse and caesarian births.

CMBFB, combined mother and baby GP consultation rate in year following birth; DCMBFB, combined mother and baby GP consultation rate in year following birth minus the mothers' consultation rate in the year preceding conception; PCMBFB, the number of postnatal mother and baby consultations as a percentage of the total mother and baby consultations:

$\mathrm{PCMBFB}=\frac{\mathrm{CMBFB}}{(\text { mothers consultations pre-conception }+ \text { CMBFB })} \times 100$

a One way analysis of variance.

${ }^{\mathrm{b}}$ Chi-squared test.

${ }^{c}$ Kruskal-Wallis test.

\section{References}

Bruijnzeels, M.A., Foets, M., van der Wouden, J.C., van den Heuvel, W.J.A. and Prins, A. 1998: Everyday symptoms in childhood: occurrence and general practitioner consultation rates. British Journal of General Practice 48, 880-84.

Carlisle, R. and Johnstone, S. 1998: The relationship between census derived socio-economic variables and general practice consultation rates in three town centre practices. British Journal of General Practice 48, 1675-78.

Carstairs, V. and Morris, R. 1989: Deprivation and mortality: an alternative to social class? Community Medicine 11, 210-19.

Chamberlain, G., Wraight, A. and Crowley, P. 1996: Home births. The report of the 1994 confidential enquiry by the National Birthday Trust Fund. Carnforth: The Parthenon Publishing Group. 
Davies, J., Hey, E., Reid, W. and Young, G. 1996: Prospective regional study of planned home births. British Medical Journal 313, 1302-306.

Foster, K., Lader, D. and Cheesbrough, S. 1997: Infant Feeding 1995. London: The Stationary Office.

Glazener, C.M.A., Abdalla, M. and Stroud, P. 1995: Postnatal maternal morbidity: extent, causes, prevention and treatment. British Journal of Obstetrics and Gynaecology 102, 282-87.

Gunn, J., Lumley, J., Young, D. 1996: Visits to medical practitioners in the first 6 months of life. Journal of Paediatrics and Child Health 32, 162-6.

Haynes, R. 1991: Inequalities in health and health service use: evidence from the General Household Survey. Social Science and Medicine 33, 361-68.

Howie, P.W., Forsyth, J.S., Ogston, S.A., Clark, A. and Florey, C.D. 1990: Protective effect of breast feeding against infection. British Medical Journal 300, 11-16.

Kumar, R. and Robson, K.M. 1984: A prospective study of emotional disorders in childbearing women. British Journal of Psychiatry 144, 35-47.
MacArthur, C., Mewis, M. and Knox, E.G. 1991: Health After Childbirth. London: HMSO.

Macfarlane, A. and Mugford, M. 2000: Birth Counts. Statistics of Pregnancy and Childbirth, 2nd edn. London: The Stationary Office.

O'Hara, M.W. and Swain, A.M. 1996: Rates and risk of postnatal depression - a meta-analysis. International Review of Psychiatry 8, 37-54.

Royal College of General Practitioners, Office of Population Censuses and Surveys, and Department of Health. 1995: Morbidity Statistics from General Practice. Fourth National Study 19911992. London: HMSO.

Saxena, S., Majeed, A. and Jones, M. 1999: Socio-economic differences in childhood consultation rates in general practice in England and Wales: prospective cohort study. British Medical Journal 318, 642-46.

Van Den Bosch, W.J.H.M., Huygen, F.J.A., Van Den Hoogen, H.J.M. and Van Weel, C. 1992: Morbidity in early childhood: differences between girls and boys under 10 years old. British Journal of General Practice, 42, 366-9. 